\title{
Estrategia de contratación de personal en las empresas mixtas petroleras venezolanas
}

\author{
Staff hiring strategy in venezuelan mixed oil companies
}

\begin{tabular}{l|r}
\cline { 2 - 2 } & Elizabeth Gutiérrez \\
\hline $\begin{array}{l}\text { Artículo recibido en septiembre } 2019 \\
\begin{array}{l}\text { Arbitrado en octubre } 2019 \\
\text { Publicado en enero } 2020\end{array}\end{array}$ & elizabethgutierrez1607@gmail.com \\
ORCID: 0000-0002-0872-0390 \\
Universidad del Zulia - Venezuela
\end{tabular}

RESUMEN

Palabras clave:
El objetivo de este estudio fue analizar las estrategias de contratación de personal en las empresas mixtas petroleras venezolanas. Metodológicamente se tipificó como descriptiva, con diseño no experimental, transeccional y de campo. La población quedó constituida por tres (3) empresas mixtas ubicadas en la Costa Oriental del Lago. La técnica de recolección de datos fue la encuesta, y el instrumento un cuestionario con 12 reactivos bajo una escala tipo Lickert. Se utilizó el juicio de expertos para la validez del mismo, y el coeficiente Alfa de Cronbach para su confiabilidad, cuyo resultado fue de 0,9214. Se aplicó como técnica para el análisis de datos el análisis estadístico descriptivo, con el cálculo de la media aritmética (X). Se evidenció que su mayor fortaleza es la selección del personal catalogada con alta aplicación; mostrando oportunidades de mejora en lo referente a las estrategias de reclutamiento del personal y de compensación.

Compensación, empresas mixtas petroleras, estrategias de contratación de personal, reclutamiento del personal, selección del personal

ABSTRACT $\mid$ The objective of this study was to analyze the personnel hiring strategies in Venezuelan mixed oil companies. Methodologically it was typified as descriptive, with a non-experimental, transectional and field design. The population was made up of three (3) mixed companies located on the eastern shore of the lake. The data collection technique was the survey, and the instrument was a questionnaire with 12 items under a Lickert-type scale. Expert judgment was used for its validity, and the Cronbach's alpha coefficient for its reliability, whose result was 0.9214. Descriptive statistical analysis was applied as a technique for data analysis, with the calculation of the arithmetic mean $(X)$. It was evident that its greatest strength is the selection of personnel classified with high application; showing opportunities for improvement in terms of staff recruitment and compensation strategies.

Compensation, mixed oil companies, personnel recruitment strategies, personnel recruitment, personnel selection 


\section{Keywords:}

\section{INTRODUCCIÓN}

El mundo de hoy es una sociedad compuesta por organizaciones, donde todas las actividades dirigidas hacia la producción de bienes $y / o$ prestación de un servicio deben ser planteadas coordinadas $y$ controladas. De allí que, las organizaciones deben definir estrategias que se anticipen a los cambios y las mantengan en el tiempo. Además, como consecuencia de la globalización y la apertura comercial, dichas estrategias deben ser sensibles a incluir en ellas la innovación tecnológica; los sectores empresariales, deben ser capaces de manejar de forma rápida y eficaz las innovaciones en materia de estrategia, en la cual se requiere generar nuevas tecnologías para la creación de productos o servicios que distinguen a la nueva manera de competir.

En ese marco, deben tomarse muchas decisiones estratégicas de operaciones, las principales decisiones están vinculadas a: productos o servicios, proceso, tecnología, capacidad, localización, calidad, personal, planificación, control y aprovisionamiento. Las empresas poseen como característica fundamental el añadir valor por medio de sus procesos, que culminan en un producto o servicio al que el consumidor encuentra cierta funcionalidad $\mathrm{o}$ utilidad. Una consecuencia de ello, es eliminar todos aquellos procesos que no añaden valor y no contribuyen positivamente al servicio final prestado al cliente.

Ahora bien, la estrategia es definida por Heizer y Render (2009), como el plan diseñado para alcanzar la misión, mientras que David (2008) la asume como los medios por los cuáles se lograrán los objetivos a largo plazo. Por lo cual, desarrollar una estrategia requiere que la empresa analice el entorno que enfrenta, de manera de obtener mejores resultados, alcanzado los objetivos propuestos.

Lo antes mencionado, supera los supuestos del pasado en el cual la producción era considerada una actividad neutral, desde una perspectiva estratégica, donde la eficiencia era el objetivo clave y la reducción de costos una manifestación operativa. De esta manera, la mayor parte de las empresas no le concedían relevancia como fuente de ventaja competitiva (Fernández y otros, 2003).

Desde una perspectiva más contemporánea, la función de producción constituye un área clave de la organización para responder de manera efectiva y distintiva a las crecientes necesidades, deseos y expectativas de los clientes, para lo cual es necesario diseñar, formular y ejecutar estrategias de producción adecuadas y pertinentes. 
Al respecto, se hace evidente la necesidad de contar con un sistema de producción integrado, desafiando el concepto tradicional enfocado en técnicas y funciones, que no guardan ninguna relación con el entorno de la empresa. De esta manera, se presenta una interacción donde cada decisión de la organización pasa a ser una decisión de operaciones, que satisface requerimientos y necesidades de las áreas 
funcionales, aprovechando a su vez las fortalezas y competencias de la organización.

En este sentido, el grado de funcionamiento de una organización y su respuesta precisa a la incertidumbre del entorno, está relacionado con el papel que juega operaciones en la toma de decisiones. Es por ello que aparece la estrategia de operaciones, que según Krajewskiy Ritzman (2008), es una visión de la función de operaciones, la cual se debe integrar con la estrategia empresarial y debe dar como resultado un patrón consistente de toma de decisiones de las operaciones y una ventaja competitiva para la compañía.

Por su parte, Skinner (2010) considera que, otra de las estrategias que deben enfrentar los administradores de operaciones se refieren a las decisiones sobre el personal, en efecto, cada vez más existen mayor cantidad de estudios en empresas en todo el mundo que destacan la influencia de las personas en la rentabilidad de las empresas, ya que el recurso humano es un factor significativo en la toma de decisiones estratégicas, y es el que se encarga de dirigir y ejecutar las operaciones de la organización.

Es por ello, que las estrategias de contratación que se llevan a cabo en la mayoría de las empresas, según Duran (2013), han evolucionado paralelamente a los avances tecnológicos y las nuevas formas de trabajar en los departamentos de RRHH. No hace falta retroceder muchos años para describir el inicio de los procesos de contratación como una evaluación de talento, candidatos o perfiles según las experiencias y los estudios expuestos en un currículum. Afirma la autora citada que, la realidad de los procesos de reclutamiento ha cambiado $\mathrm{y}$, en consecuencia, también lo han hecho las estrategias de contratación. Ya no sólo se evalúan aspectos descriptivos de la formación y la experiencia de un talento. Ahora se consideran tanto factores personales y de manera de ser del candidato como características de la organización, aspectos del puesto requerido o la proyección que tiene ese puesto en la empresa.

Estos son sólo algunos aspectos a considerar para llevar a cabo una contratación eficaz y estratégica dentro de una empresa. Los entornos laborales tienden a ser cada día más cercanos al aprendizaje continuo, independiente y autodidacta. El conocimiento, las habilidades y las competencias, ya sean innatas o aprendidas, ejercen su papel en todo tipo de entornos. Para profundizar un poco en esta afirmación, continua afirmando Duran (2013), es necesario considerar que el cambio en el modelo de reclutamiento y selección de personal ha pasado de una descripción superficial y generalizada a una forma individualizada y concreta. Todos los individuos son únicos, tienen características que los diferencian $y$, a su vez, cuentan con habilidades y competencias que los potencian y les permiten llevar a cabo de una manera $u$ otra roles, tareas y acciones.

Los expertos en materia laboral coinciden en que un individuo motivado 
puede llegar a desempeñar un rol de una manera mucho más eficaz que una persona que tiene los conocimientos técnicos adquiridos para desarrollarlo pero que carece de motivación. Esto no sucede en todos los casos, roles y tareas, pero pone de manifiesto que el talento es más productivo y competitivo cuando influyen otros factores externos a su formación y experiencia.

Por tal motivo, las habilidades $y$ competencias son clave para una contratación eficaz, logrando que las empresas consigan los objetivos planteados a través de equipos estratégicamente formados. Es importante recordar que, en temas de desarrollo personal, habilidad y competencia no son sinónimos (Duran, 2013). Las competencias engloban un conjunto de capacidades relacionadas con un puesto de trabajo concreto, mientras que las habilidades se refieren al potencial de una persona para adquirir y gestionar nociones y conocimientos nuevos.

Bajos tales afirmaciones, los gerentes de las empresas deben reconocer cada día más la importancia de involucrar a toda la organización en la toma de decisiones estratégica, para poder competir a escala mundial. Una empresa no solo compite ofreciendo nuevos productos y servicios, marketing creativo y finanzas acertadas, sino también con habilidades únicas en materia de operaciones (Krajewskiy Ritzman, 2008).

Con base a lo anteriormente expuesto, es de suma importancia la selección del personal que formará parte de la organización, según Chiavenato (2008:208) "el reclutamiento de personal es un conjunto de técnicas y procedimientos orientado a atraer candidatos potencialmente calificados y capaces de ocupar cargos dentro de la organización". Asimismo, para Invancevich y otros (2010:196), "el reclutamiento se relaciona directamente con la planeación y selección de recursos humanos, además de representar el primer contacto entre la organización y los posibles empleados". Para Duran (2013), después de definir los aspectos necesarios del perfil requerido, se lleva a cabo el proceso de reclutamiento de personal, en el cual se emplean todas las estrategias necesarias para llegar a candidatos activos y pasivos según las necesidades planteadas. Hoy es importante adecuar el reclutamiento como fuerza de en el proceso de selección de personal.

Cuando una organización determina que necesita empleados nuevos o reemplazos, puede acudir a fuentes tanto internas (asensos) como externas (personal nuevo), con el objetivo de captar al personal idóneo para cubrir las necesidades de la organización. Las ventajas que pueden derivarse del reclutamiento interno según el autor citado son: es más económico, es más rápido, presenta mayor índice de validez y seguridad, es una poderosa fuente de motivación para los empleados, aprovecha las inversiones de la empresa en entrenamiento de personal, y desarrolla un sano espíritu de competencia entre el personal.

Por otro lado, el ingreso de nuevos elementos a la empresa, según Duran (2013), 
trae consigo ventajas en la importación de ideas nuevas y diferentes enfoques acerca de los problemas internos de la empresa. Asimismo, renueva y enriquece los recursos humanos de la empresa y aprovecha las inversiones en preparación y en desarrollo del personal efectuadas por otras empresas o por ellos mismos. Igualmente, afirman Invancevich y otros (2010) que, acudir al reclutamiento externo, generalmente, absorbe más tiempo que el interno, ya que requiere la utilización de apropiadas técnicas de selección y el uso efectivo de fuentes que permitan la captación de personal.

Una vez que se dispone de un grupo idóneo de solicitantes obtenido mediante el reclutamiento, se da inicio al proceso de selección. Esta fase implica una serie de pasos que añaden complejidad a la decisión de contratar y consumen cierto tiempo. Según Invancevich y otros (2010:223), "la selección es el proceso por el que una organización escoge en una lista de solicitantes al personal que cumplen mejor con los criterios para ocupar un puesto vacante, considerando las condiciones ambientales del momento".

Asimismo para Chiavenato (2008), la selección busca solucionar dos problemas claves, como: adecuación del personal al cargo o eficiencia del personal al cargo. En general, puede decirse que el proceso selectivo debe analizarse como una comparación entre los requisitos del cargo y el perfil de los candidatos que se presentan, la cual se obtiene por medio de diferentes técnicas de selección y a través de un proceso de toma de decisión, lo cual representa la parte fundamental del proceso ya que se rechaza o se acepta a los aspirantes a ocupar el cargo vacante.

Es por ello, que el profundo cambio producido en las últimas décadas, en el rol y la importancia de las operaciones, ha traído de la mano una variación en la mirada de cómo las empresas pueden lograr ventajas competitivas. Esta disciplina ha asumido una función primordial en el desarrollo de las empresas, entendida como la dirección y el control sistemático de los procesos que transforman los insumos en productos o servicios.

Al respecto, afirma Skinner (2010), las organizaciones actuales, al estar sometidas a una intensa competencia global, necesitan, en lugar de más técnicas, un enfoque que lleve a un sistema de operaciones organizado e integrado, de manera tal, que supere al de sus competidores.

Desde esta perspectiva, las empresas del sector petrolero no escapan a esta realidad, necesitando aplicar estrategias de contratación de personal que estén alineadas entre sí, y se correspondan con la estrategia organizacional, permitiendo competir en el entorno actual regulador, económico y social. Así, estas estrategias constituyen pieza clave en el andamiaje de estas empresas; por lo tanto, para enfrentar una economía globalizada si las organizaciones esperan responder a las necesidades del país y del cliente, deben necesariamente, asumir operaciones de clase mundial. 
Aunado a lo expuesto, a nivel mundial las organizaciones más grandes son empresas petroleras, siendo el petróleo un producto esencial para muchas industrias y de vital importancia para el mantenimiento de la misma civilización industrializada, es por ello que se considera una industria crítica en la mayoría de las naciones. Al sector petrolero a nivel mundial se le confiere el carácter de base energética fundamental de la sociedad moderna, con base en el petróleo como fuente de energía con múltiple utilización, por ello la industria petrolera es considerada una de las más importantes.

Adentrando un poco más en el desarrollo del presente estudio, es necesario acotar que desde el último tercio del siglo XIX, prácticamente, todas las actividades económicas se sustentan en el petróleo como fuente energética, representando alrededor del $40 \%$ de la necesidad mundial. Dándole la importancia a la complejidad y aumento del mercado a través de un bien, con características intrínsecas, vinculadas con el acontecer de todo el planeta, como la industria petrolera.

Lo mencionado anteriormente, sostiene en Venezuela la importancia de la industria petrolera para el país, y por ende para su actividad económica. En los últimos años se ha tenido la necesidad de aumentar la cuota de producción para poder mantenerse en un mercado competitivo de manera que la economía pueda surgir. Bajo esta necesidad, en Venezuela existían 32 convenios operativos hasta el 31 de diciembre de 2005, a partir de entonces el gobierno venezolano basándose en la Ley de Hidrocarburos del año 2001 aprobada por la Asamblea Nacional decidió impulsar la migración de esta figura jurídica de convenios operativos a empresas mixtas (PDVSA, 2006).

Al respecto, las empresas mixtas son resultado de alianzas estratégicas con diferentes transnacionales, en las cuales los lineamientos relacionados con las operaciones petroleras son compartidos en beneficios de los socios del cincuenta más uno para el estado venezolano y el restante para la empresa transnacional. El modelo de empresa mixta ha traído con sigo inversiones para el país.

La ponderada repartición de las obligaciones y responsabilidades entre el accionista mayoritario y el minoritario, la participación equilibrada de ambos socios en la gobernabilidad, la toma de decisiones en el seno de la empresa mixta y el compromiso a largo plazo de ambas partes en las nuevas asociaciones, son condiciones para el fomento de la inversión y el empleo.

Ahora bien, en cuanto al ámbito de esta investigación, las empresas mixtas petroleras objeto de estudio serán las ubicadas en la Costa Oriental del Lago de Maracaibo, siendo estas Petrozamora S.A, Petrocabimas S.A y Petroquiriquire S.A., las cuales incluyen campos petroleros que cuentan con pozos productores y estaciones de flujo para su procesamiento y tratamiento.

Estas empresas, a juicio de la investigadora por ser parte activa de las mismas, trabajan bajo la influencia de los 
cambios políticos y el escenario económico que actualmente se presenta en el país, con un precio del barril petrolero cambiante, devaluaciones, desempleo, aplicación de políticas económicas destinadas a asegurar y mantener las reservas internacionales, que ponen en riesgo las operaciones y afectan el aparato productivo de la organización.

Igualmente, se quiere acotar que, estas empresas son administradas y controladas por la Corporación Venezolana de Petrolero (CVP) cuya función es maximizar el valor de los hidrocarburos del Estado Venezolano en función del bienestar colectivo. Asimismo, dichas organizaciones, deben estar alineadas con el plan siembra petrolera, en el cual están trazadas la directrices de la política energética de Venezuela hasta el año 2030, el cual comprende seis (6) grandes proyectos de desarrollo y consta de dos (2) etapas: una ejecutada entre el período 20052012, y la segunda, llevándose adelante en la etapa comprendida entre 2012 al 2030.

Por consiguiente, surge la importancia de analizar las estrategias de contratación de personal, que ayuden a las empresas a mantener la capacidad e incrementar la producción a bajo costo y con pocos recursos, con el objetivo de estar preparados para los cambios de la demanda de petróleo a nivel mundial, generando gran impacto sobre la efectividad de las empresas y alcanzar, a su vez, los objetivos propuestos por la mismas.

\section{MATERIALES Y MÉTODO}

Metodológicamente la investigación se tipificó como descriptiva, con diseño no experimental, transeccional y de campo. La población quedó constituida por 36 sujetos informantes: gerentes, superintendentes y supervisores, que trabaja en las tres (3) empresas mixtas petroleras venezolanas, específicamente las ubicadas en la Costa Oriental del Lago, siendo estas: Petrozamora S.A, Petrocabimas S.A y Petroquiriquire S.A.

La técnica de recolección de datos fue la encuesta, y el instrumento un cuestionario con 12 reactivos bajo una escala tipo Lickert, representada de la siguiente manera: Totalmente de acuerdo (Ta), De acuerdo (Da), Ni de acuerdo ni en desacuerdo (Ni), En desacuerdo (Ed), y Totalmente en desacuerdo (Td). Se utilizó el juicio de expertos para la validez del mismo, y el coeficiente Alfa de Cronbach para su confiabilidad, cuyo resultado fue de 0,9214, mostrando muy alta confiabilidad.

Se aplicó como técnica para el análisis de datos el análisis estadístico descriptivo, con el cálculo de la media aritmética $(X)^{\top}$, bajo la interpretación del baremo correspondiente, mostrado en el tabla 1. 
Tabla 1. Baremo para la interpretación de la media aritmética

\begin{tabular}{|c|c|c|}
\hline INTERVALO & CATEGORÍA & DESCRIPCIÓN \\
\hline $4.21-5.00$ & Muy alta aplicación & $\begin{array}{l}\text { Indica muy alta aplicación de las estrategias } \\
\text { de contratación de personal }\end{array}$ \\
\hline $3.41-4.20$ & Alta aplicación & $\begin{array}{l}\text { Indica alta aplicación de las estrategias de } \\
\text { contratación de personal }\end{array}$ \\
\hline $2.61-3.40$ & Moderada aplicación & $\begin{array}{l}\text { Indica moderada aplicación de las } \\
\text { estrategias de contratación de personal }\end{array}$ \\
\hline $1.81-2.60$ & Baja aplicación & $\begin{array}{l}\text { Indica baja aplicación de las estrategias de } \\
\text { contratación de personal }\end{array}$ \\
\hline $1.00-1.80$ & Muy baja aplicación & $\begin{array}{l}\text { Indica muy baja aplicación de las estrategias } \\
\text { de contratación de personal }\end{array}$ \\
\hline
\end{tabular}

Fuente: Elaboración propia (2020)

RESULTADOS Y DISCUSIÓN

Los valores presentados en la tabla 2, corresponden al indicador reclutamiento del personal. En los mismos se aprecia como la media se ubicó en 2,88 indicando la existencia de moderada aplicación de las actividades y procesos asociados al mismo, es decir, en las empresas sometidas a este estudio, el reclutamiento del personal como parte de la estrategia de contratación de personal se aplica de manera moderada.

Tal resultado obedece al nivel de aplicación otorgado a cada ítem asociado al indicador, donde otorgaron alta aplicación $(3,08)$ al primer ítem, el cual expresa que si al momento de requerir personal para un cargo vacante se le da oportunidad al personal interno (ascensos, traslados, promociones), también obtuvo una alta aplicación $(3,83)$ en cuanto a que si poseen programas de desarrollo del personal. Sin embargo, según los encuestados, se da baja aplicación a lo concerniente a que si acuden a fuentes externas para reclutar su personal $(2,08)$ y si tienen asociaciones con instituciones externas para el reclutamiento de personal (universidades, centros de capacitación u otros centros gremiales) obtuvo un 2,50 . 
Tabla 2. Indicador: Reclutamiento del personal

\begin{tabular}{|c|c|c|c|}
\hline & ÍTEM & & \\
\hline & resa mixta donde usted labora: & $\boldsymbol{X}$ & CATEGORIA \\
\hline 1. & $\begin{array}{l}\text { Al momento de requerir personal para un cargo } \\
\text { vacante se le da oportunidad al personal interno } \\
\text { (ascensos, traslados, promociones). }\end{array}$ & 3,08 & Alta aplicación \\
\hline 2. & Poseen programas de desarrollo de personal. & 3,83 & Alta aplicación \\
\hline 3. & Acuden a fuentes externas para reclutar su personal. & 2,08 & Baja aplicación \\
\hline 4. & $\begin{array}{l}\text { Tiene asociaciones con instituciones externas para el } \\
\text { reclutamiento de personal (universidades, centros de } \\
\text { capacitación u otros centros gremiales). }\end{array}$ & 2,50 & Baja aplicación \\
\hline & ador: & 2,88 & Moderada aplicación \\
\hline
\end{tabular}

Fuente: Elaboración propia (2020)

De acuerdo a estos resultados, se valida medianamente lo indicado por Duran (2013), para quien, el reclutamiento de personal es un sistema de información, mediante el cual la empresa divulga y ofrece al mercado de recursos humanos las oportunidades de empleo que pretende llenar. Para ser eficaz, el reclutamiento debe atraer una cantidad de candidatos suficiente para abastecer de modo adecuado el proceso de selección. Cuando una organización determina que necesita empleados nuevos o reemplazos, puede acudir a fuentes tanto internas como externas, con el objetivo de captar al personal idóneo para cubrir las necesidades de la organización.

De igual forma, ambas fuentes deben ser factibles de aplicarse en la práctica de las empresas mixtas bajo estudio, ya que se puede presentar la oportunidad de promocionar a los trabajadores internos o la necesidad de captar personal externo en la organización. Sin embargo, en la fuente externa están mostrando oportunidades de mejora al considerarlas con baja aplicación (ítems 3 y 4).

De seguido, en la tabla 3 se muestra la información correspondiente al indicador selección del personal; según estos datos, se aprecia para el indicador una media de 3,95 indicando alta aplicación de la selección del personal como parte de la estrategia de contratación de personal aplicada en las empresas bajo estudio.

De tal forma, se aplica de manera muy alta el que verifiquen la documentación o referencias del personal $(4,42)$; de forma alta si tienen entrevistas preestablecidas para la selección del personal $(4,11)$; y si realizan pruebas de conocimientos o capacidad de estudio a los candidatos $(3,94)$; no obstante, califican con moderada aplicación el que realicen comparación entre los requisitos del cargo y el perfil de los candidatos al momento de la selección del personal $(3,33)$. 
Tabla 3. Indicador: Selección del personal

\begin{tabular}{|c|c|c|c|}
\hline \multicolumn{2}{|r|}{ ÍTEM } & \multirow[b]{2}{*}{$\overline{\boldsymbol{X}}$} & \multirow[b]{2}{*}{ CATEGORÍA } \\
\hline \multicolumn{2}{|r|}{ En la empresa mixta donde usted labora: } & & \\
\hline 5. & $\begin{array}{l}\text { Realizan comparación entre los requisitos del cargo y el } \\
\text { perfil de los candidatos al momento de la selección del } \\
\text { personal. }\end{array}$ & 3,33 & Moderada aplicación \\
\hline 6. & $\begin{array}{l}\text { Tienen entrevistas preestablecidas para la selección del } \\
\text { personal. }\end{array}$ & 4,11 & Alta aplicación \\
\hline 7. & $\begin{array}{l}\text { Realizan pruebas de conocimientos o capacidad de } \\
\text { estudio a los candidatos. }\end{array}$ & 3,94 & Alta aplicación \\
\hline \multirow[t]{2}{*}{8.} & Verifican la documentación o referencias del personal. & 4,42 & Muy alta aplicación \\
\hline & Total indicador: & 3,95 & Alta aplicación \\
\hline
\end{tabular}

Fuente: Elaboración propia (2020)

Con base en estos resultados, se valida lo indicado por Invancevich y otros (2010), para quienes la selección es el proceso por el que una organización escoge en una lista de solicitantes al personal que cumplen mejor con los criterios para ocupar un puesto vacante, considerando las condiciones ambientales del momento.

Sin embargo, validan medianamente a estos autores cuando afirman que, el proceso selectivo debe analizarse como una comparación entre los requisitos del cargo y el perfil de los candidatos que se presentan, la cual se obtiene por medio de diferentes técnicas de selección y a través de un proceso de toma de decisión, lo cual representa la parte fundamental del proceso ya que se rechaza o se acepta a los aspirantes a ocupar el cargo vacante.

De manera similar, también se logra coincidencia con lo expuesto por la investigadora cuando afirma que el proceso de selección es la fase inmediatamente siguiente al proceso de reclutamiento, en donde la empresa debe seleccionar de lista de solicitantes al personal que cumpla mejor con los criterios para ocupar el puesto vacante. Esta selección debe realizarse con base a los aspectos del trabajo a desempeñar, se deben realizar exámenes psicotécnicos, y se deben evaluar las habilidades $y$ competencias de los candidatos.

Con relación al último indicador de esta dimensión denominado compensación, la tabla 4 muestra la información recabada para el mismo. Se observa una media de 3,21, implicando moderada aplicación de las actividades asociadas a la compensación como parte de la estrategia de contratación de personal aplicada en las empresas bajo estudio. 
Tabla 4. Indicador: Compensación

\begin{tabular}{|c|c|c|c|}
\hline & \multirow{3}{*}{$\begin{array}{c}\overline{\boldsymbol{X}} \\
3,19\end{array}$} & \multirow{3}{*}{$\begin{array}{l}\text { CATEGORÍA } \\
\text { Moderada aplicación }\end{array}$} \\
\hline \multicolumn{2}{|c|}{$\begin{array}{c}\text { ÍTEM } \\
\text { En la empresa mixta donde usted labora: }\end{array}$} & & \\
\hline 9. & $\begin{array}{l}\text { La remuneración salarial es similar en relación a otras } \\
\text { empresas del sector. }\end{array}$ & & \\
\hline 10. & $\begin{array}{l}\text { Se rigen por políticas del gobierno para definir sus } \\
\text { salarios. }\end{array}$ & 3,33 & Moderada aplicación \\
\hline 11. & Utilizan guías administrativas para aumentos salariales. & 3,22 & Moderada aplicación \\
\hline 12. & $\begin{array}{l}\text { Utilizan tabuladores para la asignación de salarios en } \\
\text { función de las experiencias del trabajador. }\end{array}$ & 3,08 & Moderada aplicación \\
\hline
\end{tabular}

Total indicador: 3,21 Moderada aplicación

Fuente: Elaboración propia (2020)

El valor obtenido en la media, es producto de la moderada aplicación otorgada a todos los ítems utilizados, donde se les consultaba si: la remuneración salarial es similar en relación a otras empresas del sector $(3,19)$; se rigen por políticas del gobierno para definir sus salarios $(3,33)$; utilizan guías administrativas para aumentos salariales $(3,22)$, y utilizan tabuladores para la asignación de salarios en función de las experiencias del trabajador $(3,08)$.

Corresponde este resultado, medianamente, a lo que describe Chiavenato (2008), para quien los salarios deben tener un equilibrio tanto interno como externo; cuando se habla interno, el salario debe ser equitativo y justo para toda la organización, y el mismo se logra mediante la evaluación y clasificación de los cargos a través de la política salarial de la empresa. Las políticas salariales deben contener, estructuras de cargos y salarios, salarios de admisión para las diversas clases salariales y previsión de reajustes. En cuanto a equilibrio externo, se basa en la investigación salarial, de manera que tenga relación con otras empresas similares que actúen en el mercado de trabajo.

También se valida la posición de la investigadora, para quien la remuneración salarial debe ser equitativa tanto dentro de la organización como en comparación con el mercado, con el objetivo de lograr trabajadores conformes y motivados. No obstante, se acota que, en el mercado laboral de Venezuela, los salarios están regidos por tabuladores de sueldos y salarios para los profesionales y no profesionales, tomando en consideración el salario mínimo, el cual es establecido por el Ejecutivo Nacional y está estipulado en la Ley Orgánica del Trabajo en el Art. 167.

Seguidamente, en la tabla 5, se resume el comportamiento de la dimensión estrategia de contratación de personal, con sus respectivos indicadores. En ésta, se aprecia 
que el valor para la media se ubicó en 3,34, indicando moderada aplicación de la estrategia de contratación de personal como parte de las estrategias de operaciones que se aplican en las empresas mixtas petroleras venezolanas.

Tabla 5. Dimensión: Estrategia de contratación de personal

\begin{tabular}{lcl}
\hline \multicolumn{1}{c}{ INDICADOR } & $\overline{\boldsymbol{X}}$ & \multicolumn{1}{c}{ CATEGORÍAS } \\
\hline Reclutamiento del personal & 2,88 & Moderada aplicación \\
Selección del personal & 3,95 & Alta aplicación \\
Compensación & 3,21 & Moderada aplicación \\
Resumen dimensión & $\mathbf{3 , 3 4}$ & Moderada aplicación \\
\hline
\end{tabular}

Fuente: Elaboración propia (2020)

Este resultado, de moderada aplicación para la estrategia de contratación de personal, es determinado por el nivel de aplicación alcanzado para cada uno de sus indicadores; donde la selección del personal se ubicó en alta aplicación (3,95); sin embargo, tanto el reclutamiento del personal $(2,88)$ como la compensación $(3,21)$ evidenciaron aplicación moderada.

Estos resultados validan, medianamente, a Skinner (2010) para quien otra de las estrategias que deben enfrentar los administradores de operaciones se refieren a las decisiones sobre el personal, en efecto, cada vez más existen mayor cantidad de estudios en empresas en todo el mundo que destacan la influencia de las personas en la rentabilidad de las empresas, ya que el recurso humano es un factor significativo en la toma de decisiones estratégicas, y es el que se encarga de dirigir y ejecutar las operaciones de la organización.

De igual forma, validan a la investigadora cuando afirma que, el llevar a cabo una estrategia de contratación exitosa involucra inferir el desempeño de un candidato ante un puesto de trabajo específico, considerando su proceso continuo de aprendizaje y desarrollo en términos de autosuficiencia y adaptación a nuevos escenarios, sin que su formación previa afecte. En consecuencia, hoy día se necesita de procesos de reclutamiento de personal que incluyan evaluaciones de los candidatos centradas en su desempeño y en la adquisición de nuevos conocimientos en diversas condiciones.

\section{CONCLUSIONES}

Al analizar las estrategias de contratación de personal en las empresas mixtas petroleras venezolanas, se evidencio que su mayor fortaleza es la selección del personal catalogada con alta aplicación; mostrando oportunidades de mejora en lo referente a las estrategias de reclutamiento del personal y de compensación. De tal manera, que es importante: 
- Mejorar el programa de capacitación y desarrollo del personal, de manera que garantice que todo el personal reciba los cursos planificados anualmente, generando un talento humano mayor preparado.

- Profundizar las estrategias de contratación y futuras técnicas que permitan seleccionar y contratar al personal adecuado.

- Realizar revisión de las descripciones de cargos establecidos, de modo de actualizarlos existentes y desarrollar aquellos que no se encuentren, permitiendo que el personal conozca las funciones que deben desarrollar para alcanzar los objetivos propuestos por la organización, igualmente ayuda al proceso de selección de personal nuevo.

- Realizar planes de adecuación salarial, que permita crear e implementar cada periodo de tiempo, mejoras en los beneficios de los trabajadores, de manera que se logre recompensar por los logros obtenidos.

\section{REFERENCIAS}

Chiavenato, I. (2008). Introducción a la teoría general de la administración. Quinta Edición. Editorial McGraw-Hill. España

David, F. (2008). Conceptos de Administración Estratégica. 6ta edición. Editorial Prentice Hall. México

Duran, A. (2013). Estrategias de Contratación: Habilidades y Competencias. Blog de reclutamiento 2.0. Disponible en: http://blog.talentclue.com/bid/322802/Es trategias-de-Contrataci-n-Habilidades-y Competencias

Fernández E.; Avellana, L. y Fernández, M. (2003). Estrategia de producción. Segunda edición. Editorial McGraw-Hill Interamericana de México, México

Heizer, J. y Render B. (2009). Dirección de la producción y de operaciones. Decisiones estratégicas. Editorial Pearson. Prentice Hall. México

Ivancevich, J., Konopaske, R., Matteson M. y Núñez, J. (2010) Comportamiento organizacional y administración de empresas. Décima edición. Editorial McGraw-Hill Interamericana de España S.L. España

Krajewski, L. y Ritzman, L. (2008). Administración de operaciones: estrategia y análisis. Séptima edición. Editorial Pearson Educación, S.A. México

PDVSA (2006). Serie Plena Soberanía Petrolera $\mathrm{N}^{\circ} 4$. Empresas mixtas al servicio del pueblo. http://www.pdvsa.com/2006

Proyecto de Ley de Reforma de la Ley Orgánica del Trabajo" (2004) (Aprobado en $1^{\circ}$ discusión el 17-06-2003). Gaceta Laboral. Vol.10, $\mathrm{N}^{\circ} 1 . \quad$ Disponible en: http://www.serbi.luz.edu.ve/scielo.php ?Script =sci_arttext \& pid=S131585972004004000006 \& Ing=es \& nrm=iso.ISSN1315-8597

Skinner, W. (2010). Administración estratégica de operaciones. Editorial McGraw-Hill. México 